\title{
Digital immunohistochemistry wizard: image analysis-assisted stereology tool to produce reference data set for calibration and quality control
}

Benoît Plancoulaine ${ }^{1 *}$, Aida Laurinaviciene ${ }^{2,3}$, Raimundas Meskauskas ${ }^{2,3}$, Indra Baltrusaityte ${ }^{2,3}$, Justinas Besusparis ${ }^{2,3}$, Paulette Herlin², Arvydas Laurinavicius ${ }^{2,3}$

From 12th European Congress on Digital Pathology

Paris, France. 18-21 June 2014

\begin{abstract}
Background: Digital image analysis (DIA) enables better reproducibility of immunohistochemistry (IHC) studies. Nevertheless, accuracy of the DIA methods needs to be ensured, demanding production of reference data sets. We have reported on methodology to calibrate DIA for Ki67 IHC in breast cancer tissue based on reference data obtained by stereology grid count. To produce the reference data more efficiently, we propose digital IHC wizard generating initial cell marks to be verified by experts.

Methods: Digital images of proliferation marker Ki67 IHC from 158 patients (one tissue microarray spot per patient) with an invasive ductal carcinoma of the breast were used. Manual data $(\mathrm{mD})$ were obtained by marking Ki67positive and negative tumour cells, using a stereological method for 2D object enumeration. DIA was used as an initial step in stereology grid count to generate the digital data (dD) marks by Aperio Genie and Nuclear algorithms. The $\mathrm{dD}$ were collected into XML files from the DIA markup images and overlaid on the original spots along with the stereology grid. The expert correction of the $\mathrm{dD}$ marks resulted in corrected data $(\mathrm{CD})$. The percentages of Ki67 positive tumour cells per spot in the $\mathrm{mD}, \mathrm{dD}$, and $\mathrm{cD}$ sets were compared by single linear regression analysis. Efficiency of $\mathrm{CD}$ production was estimated based on manual editing effort.

Results: The percentage of Ki67-positive tumor cells was in very good agreement in the $\mathrm{mD}, \mathrm{dD}$, and $c \mathrm{D}$ sets: regression of $c D$ from $d D\left(R^{2}=0.92\right)$ reflects the impact of the expert editing the $d D$ as well as accuracy of the DIA used; regression of the $\mathrm{CD}$ from the $\mathrm{mD}\left(\mathrm{R}^{2}=0.94\right)$ represents the consistency of the DIA-assisted ground truth $(\mathrm{CD})$ with the manual procedure. Nevertheless, the accuracy of detection of individual tumour cells was much lower: in average, 18 and 219 marks per spot were edited due to the Genie and Nuclear algorithm errors, respectively. The DIA-assisted $c D$ production in our experiment saved approximately $2 / 3$ of manual marking.
\end{abstract}

Conclusions: Digital IHC wizard enabled DIA-assisted stereology to produce reference data in a consistent and efficient way. It can provide quality control measure for appraising accuracy of the DIA steps.

\footnotetext{
* Correspondence: benoit.plancoulaine@unicaen.fr

'Path-Image/BioTiCla, University of Normandy, Unicaen, Caen, France

Full list of author information is available at the end of the article
} 


\section{Background}

Digital image analysis (DIA) brings great perspectives to improve reproducibility and capacity of immunohistochemistry (IHC) studies. Nevertheless, the benefits of reproducibility, capacity and clinical utility are not a substitute for accuracy or objectivity of the DIA methods established by comparison to reference data or criterion standard, preferably representing same type of data obtained by direct and more reliable measurement $[1,2]$. While validation studies in digital IHC commonly relied on pathologists' visual evaluation or biologic ground truth (e.g., HER2 FISH [3]) as a criterion standard, more recently the effort was put into manual counting of cells to obtain the reference data $[4,5]$.

We have recently reported [6] on methodology to validate and calibrate DIA for Ki67 IHC in breast cancer tissue based on reference data obtained by stereology grid count performed on the same images: comparison of the DIA results to the reference data enabled "knowledgebased" fine-tuning of the DIA settings to achieve better accuracy. Although the methodology was successful, its practical application would be time-consuming and dependent on expert human resource to produce the data. In the perspective of multiple IHC markers to be analyzed with variable IHC staining protocols and scanning platforms, the efficiency of continuous quality control and production of the data sets becomes an important prerequisite. This demand has been recognized in broad field of bio-image informatics with the statement that full-scale adoption of automated DIA tools would require efficient production and maintenance of the data sets and provision of integrated editing tools [7]. The amount of manual expert work needed on large images with a huge number of cells is a critical bottle-neck in adoption of the editingbased approaches [8]. In brain tissue analyses, sophisticated approaches have been proposed to decrease the workload by calculating a segmentation confidence score for each cell [9] or identifying potential outliers to prioritize the expert review [10].

Clinical adoption of digital IHC techniques could be synergized by tools enabling efficient production of reference data sets for divergent IHC applications requiring proper quality controls. To the best of our knowledge, editing-based approaches have not been developed in the field of digital IHC. We therefore present a technique (dIHC Wizard) which combines the swiftness of image processing and the unbiased approach of stereology and which aims at alleviating manual workload of the expert in obtaining reference data sets. DIA is used as an initial step in stereology grid count procedure to reduce the need for manual work. The DIA-generated data $(\mathrm{dD})$ are then edited by an expert to produce the corrected data $(\mathrm{cD})$ which can be regarded as quality-assured data and be used as the standard criterion for further DIA calibrations.

\section{Methods}

Digital images of Ki67 IHC from 158 female patients (one millimeter-diameter tissue microarray (TMA) spot per patient) with an invasive ductal carcinoma of the breast were used for the study. The study was approved by the Lithuanian Bioethics Committee. The patients' consent to participate in the study was obtained. The TMA were constructed, stained, and scanned as previously described [11]. Manual data $(\mathrm{mD})$ were obtained in our previous study [6] by marking Ki67-positive and negative tumour cell profiles, using a stereological method for 2D object enumeration [12] implemented in the Stereology module (ADCIS, France) with a test grid of systematically sampled frames overlaid on a spot image in ImageScope (Aperio Technologies, USA). Automatic image processing was performed using the combination of the Genie and Nuclear algorithms from Aperio Technologies (Vista USA) calibrated in the previous study [6]. The Genie software is used to isolate the epithelial compartment of the breast carcinoma and discard stromal and inflammatory components. Nuclear algorithm allows the refined segmentation of individual nuclei of cancer cell profiles and the color characterization of immunostained and counterstained nuclei. The dIHC Wizard workflow is presented in the Figure 1. The $\mathrm{dD}$ marks were produced from the multicolor mask image resulting from the automatic analysis. For this purpose, an Excess Red Green Blue image was computed (2R-G-B, 2G-R-B and 2B-R-G) and fixed thresholds applied to isolate the automatically labeled immunostained and counterstained epithelial nuclear profiles (Figure 2). The $\mathrm{dD}$ marks were collected from the centroid of the labeled nuclear profiles and stored into an XML file which was then overlaid on the original spot images to generate relevant XML tags along with the stereology grid (Figure 2). This allowed the expert to edit, by manual adding or deleting, the $\mathrm{dD}$ marks, as needed to produce the $\mathrm{cD}$. The $\mathrm{mD}$ and $\mathrm{cD}$ were produced by the same experts. A new file containing $\mathrm{XML}$ tags representing the $\mathrm{cD}$ was generated and compared to the original file $(\mathrm{dD})$ to retrieve data for statistical analysis (Figure 3). The percentage of Ki67 positive tumour cell profiles per spot (Ki67\%) was used to compare the data sets. True and false positive and negative Ki67 tumor cell rates as well as lost or false tumor cells were established for each spot. The $\mathrm{mD}, \mathrm{dD}$, and $\mathrm{cD}$ sets were compared by single linear regression analysis. Agreement between individual measurements was also estimated and visualized by Bland and Altman plots [13]. Furthermore, the undetected tumor cells 


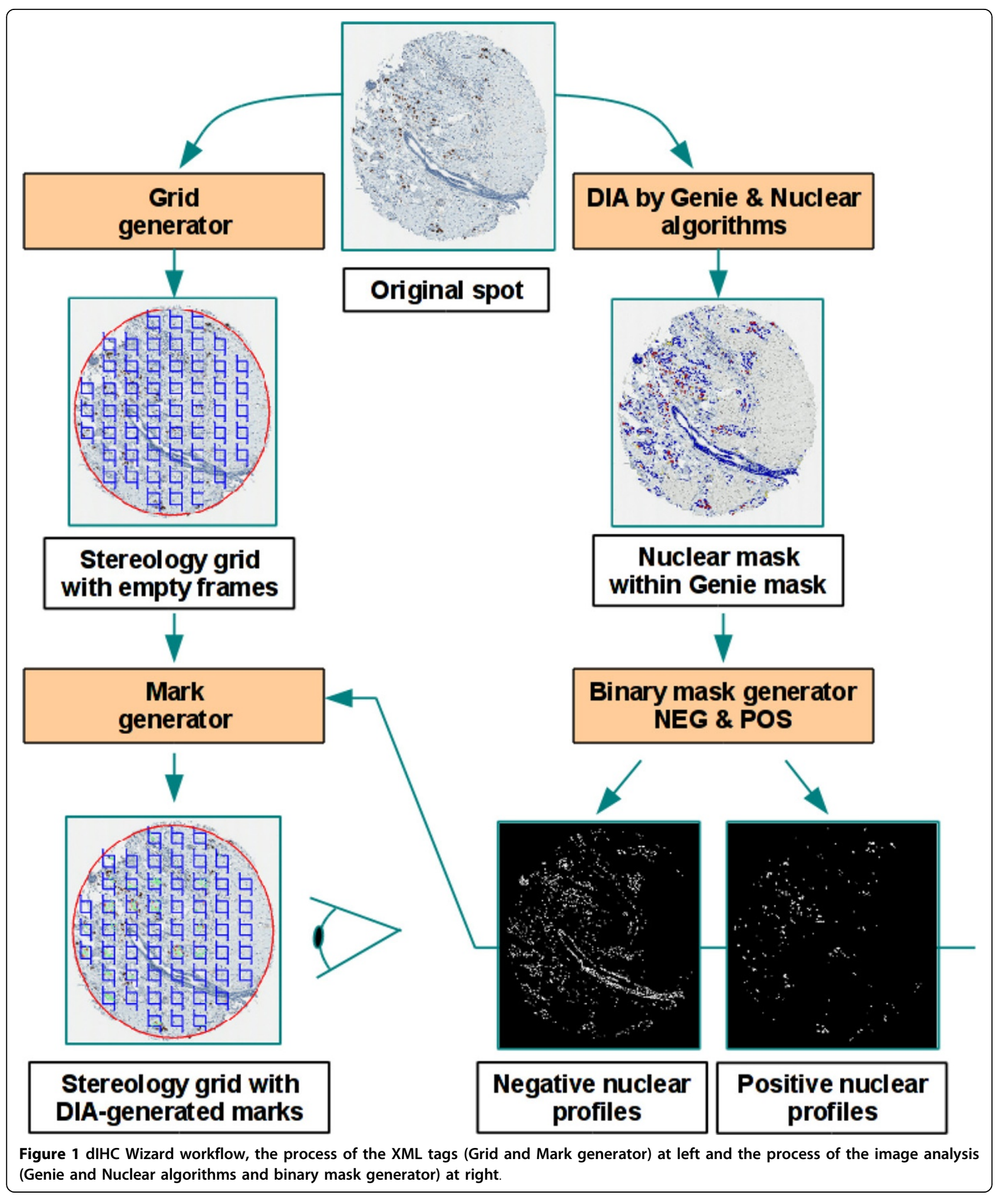

were subcategorized to "By the Genie" and "By the Nuclear" algorithms based on reference of their coordinates to the original DIA markup images. Falsely detected tumor cells were due to the Genie or the
Nuclear components. This allowed comparing the impact of the Genie and Nuclear algorithms on $\mathrm{dD}$ error rate providing insights on relative accuracy of each DIA component. Efficiency of $\mathrm{cD}$ production was 


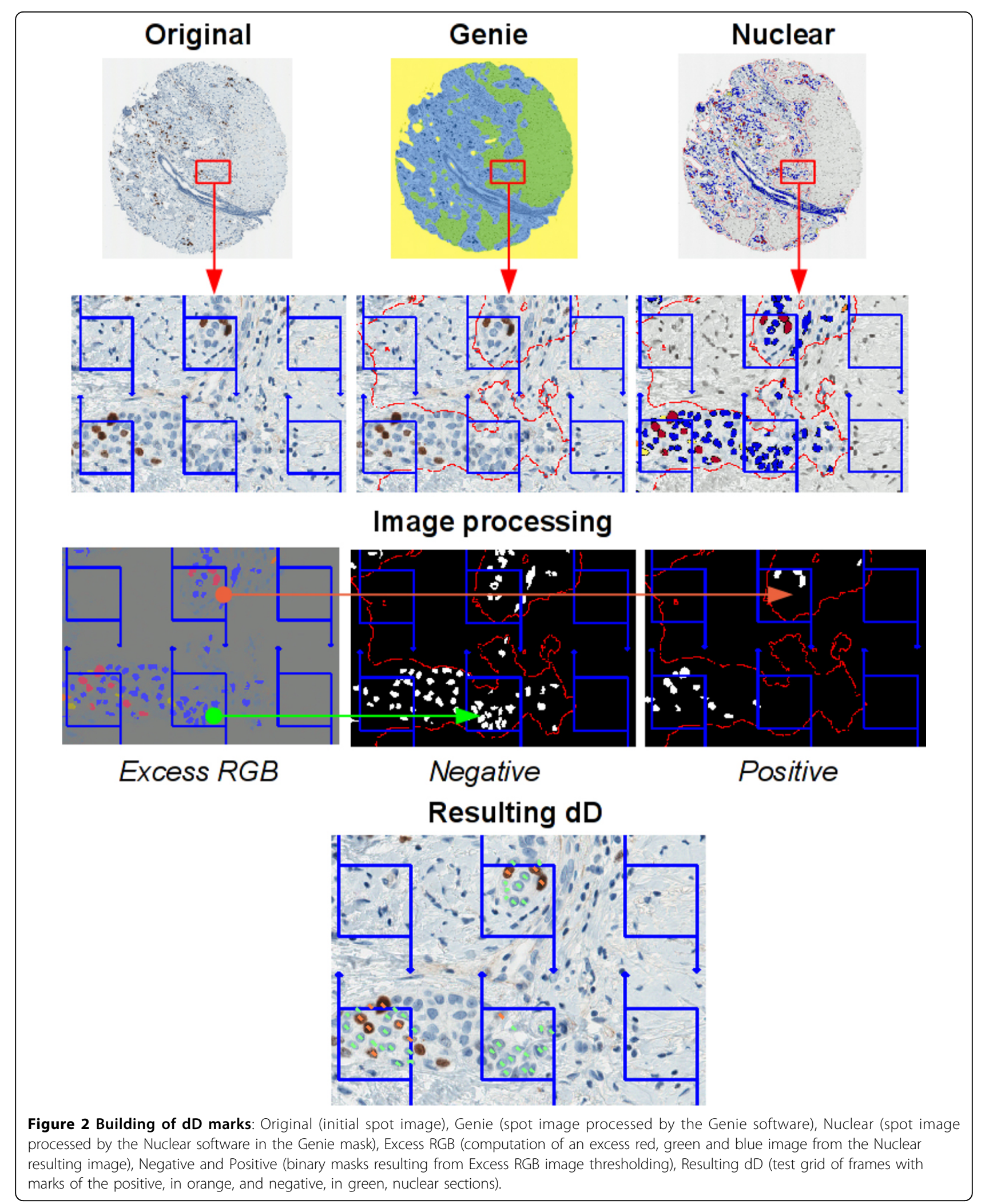




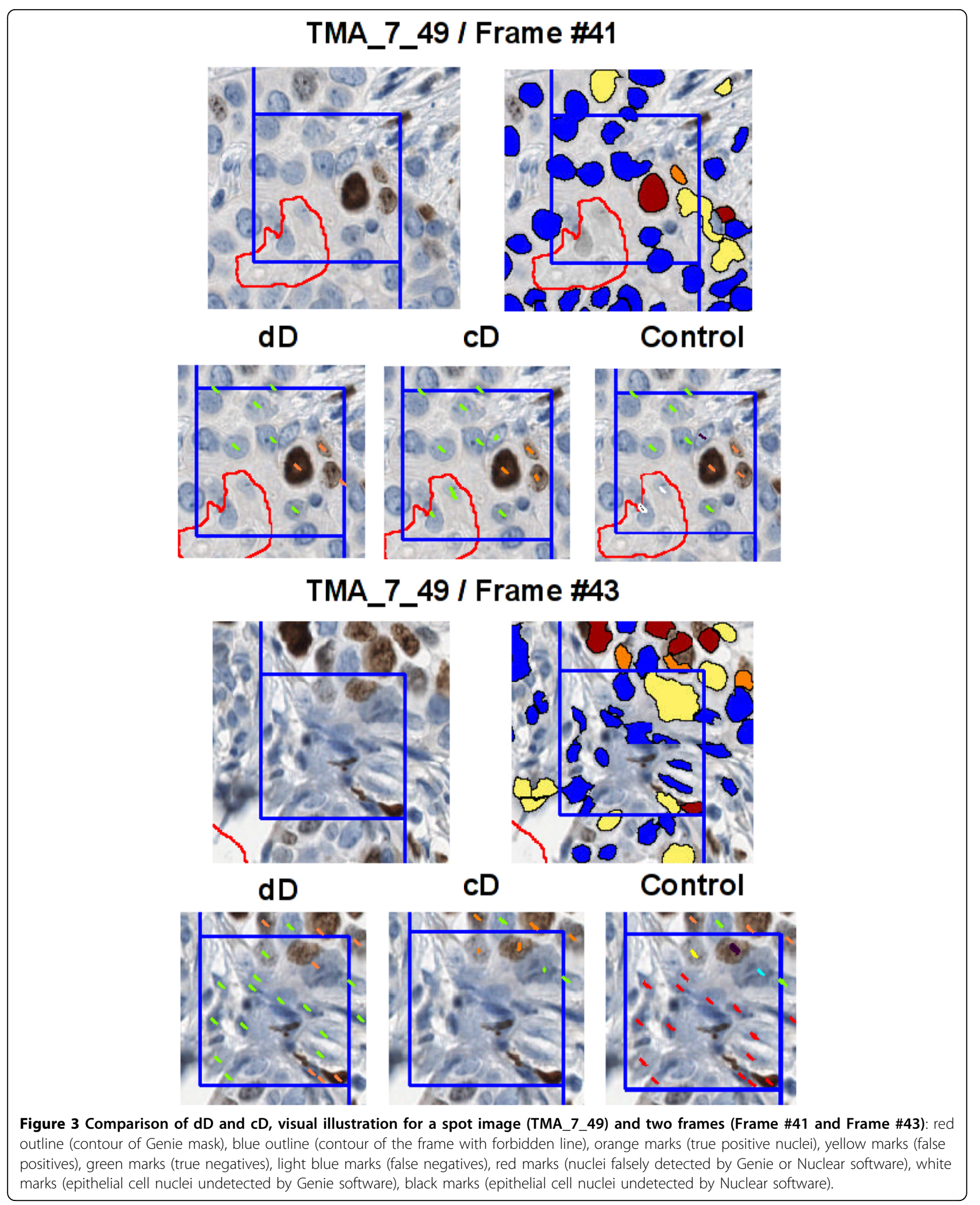


estimated based on expert editing effort required. Statistical significance level was set at $\mathrm{p}<0.05$.

\section{Results and discussion}

The percentage of Ki67-positive tumor cells in each spot image (Ki67\%) was in a very good agreement in the $\mathrm{mD}$, $\mathrm{dD}$, and $\mathrm{cD}$ sets when compared by single linear regression analysis (Figure 4). Regression of the $\mathrm{cD}$ from the $\mathrm{dD}\left(\mathrm{R}^{2}=0.92\right)$ reflects the impact of the expert editing the $\mathrm{dD}$ as well as accuracy of the DIA used to produce the $\mathrm{dD}$. Regression of the $\mathrm{cD}$ from the $\mathrm{mD}\left(\mathrm{R}^{2}=0.94\right)$ reflects the consistency of the DIA-assisted standard criterion $(\mathrm{cD})$ production with the manually obtained standard criterion. Slight bias in the opposite directions can be noted in both comparisons.

Besides the comparison of the summarized TMA spot indicators in the data sets, our method provides detailed information on accuracy of detection and the IHC-positivity interpretation by DIA of individual cells.
Despite the very good agreement of the Ki67\% per spot between the data sets, the accuracy of detection of individual tumor cells was much lower. Based on the expert corrections made on the $\mathrm{dD}$ to produce the $\mathrm{cD}$, in average 18 and 219 marks per spot were edited due to the Genie and Nuclear algorithm errors, respectively (Figure 5); the mean of marks per spot was 663 . In all 158 TMA spots, a total of 105,486 nuclei have been identified in the $\mathrm{cD}$ while 39,710 (37.6\%) expert corrections have been made on the $\mathrm{dD}$, including 2,941 (2.8\%) and 24,727 (23.4\%) edits to correct the Genie and Nuclear under-detection of epithelial nuclear profiles, respectively, and 10,793 (10.2\%) overdetection of epithelial nuclear profiles by Genie or Nuclear. As described in the Methods section, the Nuclear component of the DIA was applied only on the epithelial mask already detected by the Genie component, therefore, the performance indicators of the Nuclear detection component are "Genie-dependent".
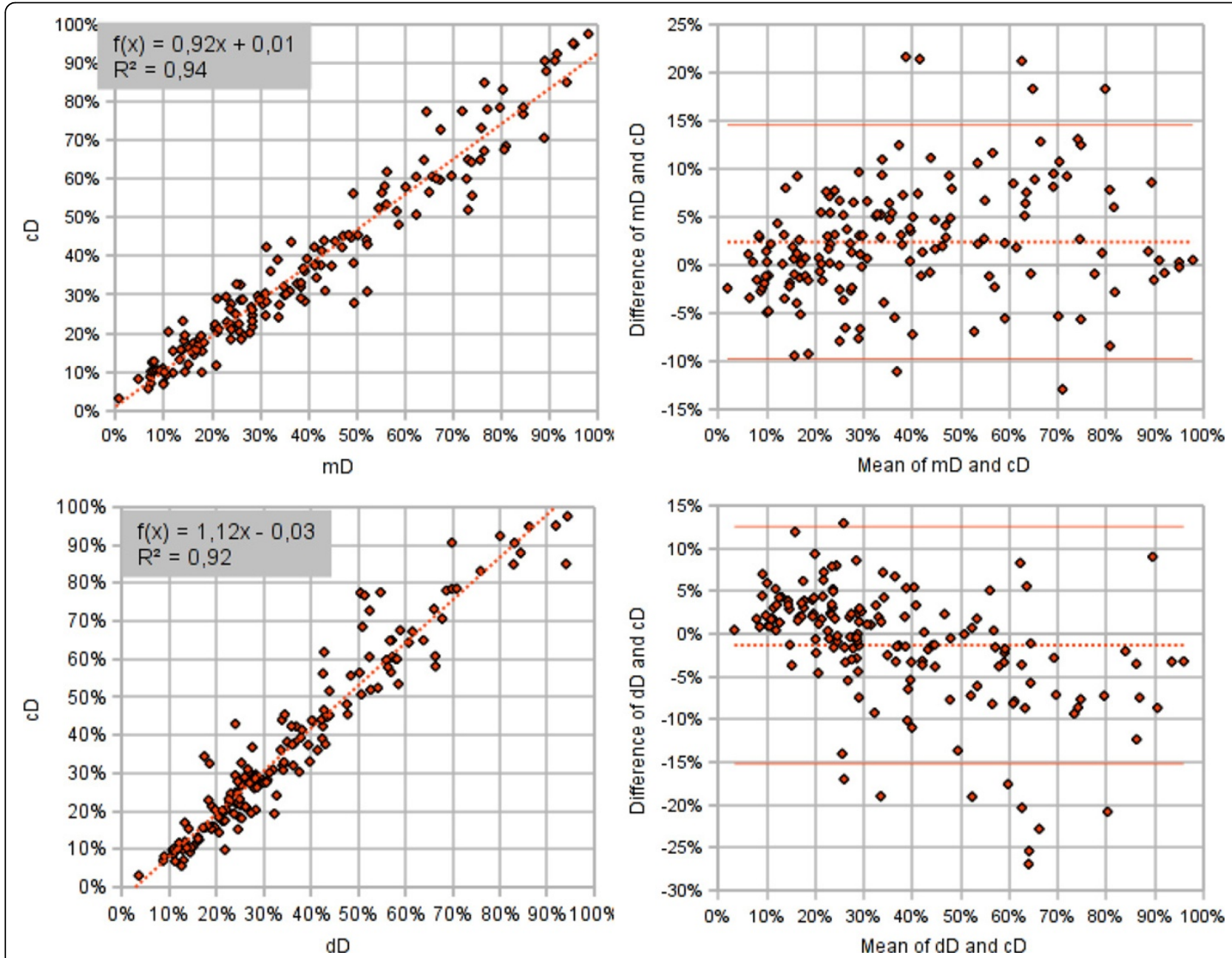

Figure 4 Upwards, comparison between $\mathrm{mD}$ and $\mathrm{CD}$ by single linear regression analysis at left and Bland Altman analysis at right; downwards, comparison between $\mathrm{dD}$ and $\mathrm{CD}$ by single linear regression analysis at left and Bland Altman analysis at right. 


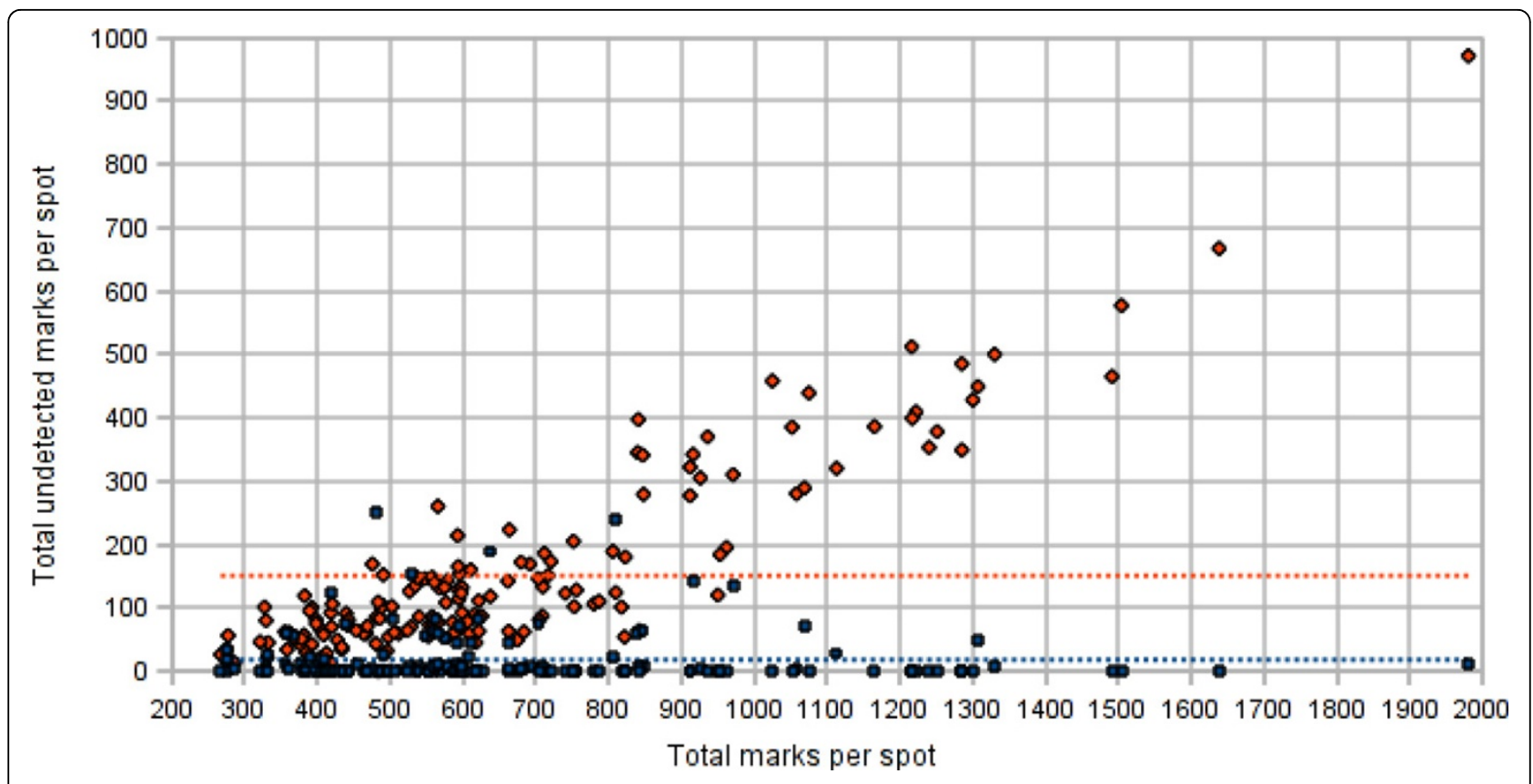

Figure 5 Numbers of tumor cell nuclei undetected by the Genie (blue dots) and the Nuclear (orange dots) algorithms.

When nucleus is properly identified, the IHC staining interpretation (Ki67-positive versus negative) by the Nuclear algorithm can be regarded as excellent: overall, only $1,035(1 \%)$ false positive and $214(0.2 \%)$ false negative tumor nuclei were corrected by the experts (Figure 6).

Importantly, our data highlight the impact of the accuracy definition and the DIA validation results: it was perfect with regard to the Ki67\% result per image which could be interpreted as sufficient for clinical use. However, the accuracy of the individual tumour cell detection was less satisfactory and may be taken as a warning sign on the road of developing robust automated DIA tools. Perhaps, different applications may require more conservative accuracy definitions and validation procedures for specific DIA tasks.

Furthermore, our approach provided a benefit of "decomposing" the accuracy of 3 DIA components used by one expert editing procedure. Importantly, the Genie component outperformed the Nuclear algorithm in terms of tumor cell detection in our experiment. We are not aware of published data on the relative impact on DIA accuracy caused by automated tumour (epithelial) tissue and tumour cell identification components. While the issue of automatic detection and segmentation of cell nuclei in histopathology images is well-addressed, accuracy of tumour tissue detection would require targeted studies. Lastly, discrimination between the Ki67 IHC staining result (positive versus negative) by the DIA was excellent in our study.

We therefore suggest that even if the accuracy of the Ki67\% (image-based estimate) on the whole spot series was very good, more conservative cell-based validation approach could uncover the "functional anatomy" of the DIA tools and point further DIA improvement efforts in the right direction to achieve most robust DIA processes and results.

The accuracy of the DIA tool used to produce the $\mathrm{dD}$ set determines the efficiency of the DIA-assisted $\mathrm{cD}$ production. In our case, the approach saved approximately $2 / 3$ of manual editing, however, the effort to review the images remained the same. The efficiency can be further increased by improving ergonomics of the stereology tool and employing a better calibrated DIA-assistance. In our experiment, we applied systematic random sampling by stereology grid; it is a simple method to control the amount of manual work, however, it is subject to variable amount of tumour tissue and cellularity in the images. This disadvantage can be compensated by automated resizing of the stereology grid based on the results of the DIA to produce the $\mathrm{dD}$ set in order to get optimal number of cells to be reviewed per image.

Last but not least, the $\mathrm{mD}$ and/or $\mathrm{cD}$ sets are qualityassured and contain information on exact location of the cells in the image, therefore, can be utilized as standard criterion templates to validate, calibrate, and train DIA tools. On a more global perspective, the reference data libraries may serve as benchmark datasets for automated DIA - the demand well-recognized and addressed in computational neuroscience and bioimage informatics, in general, with tasks of much higher complexity than the digital IHC $[14,15]$. 

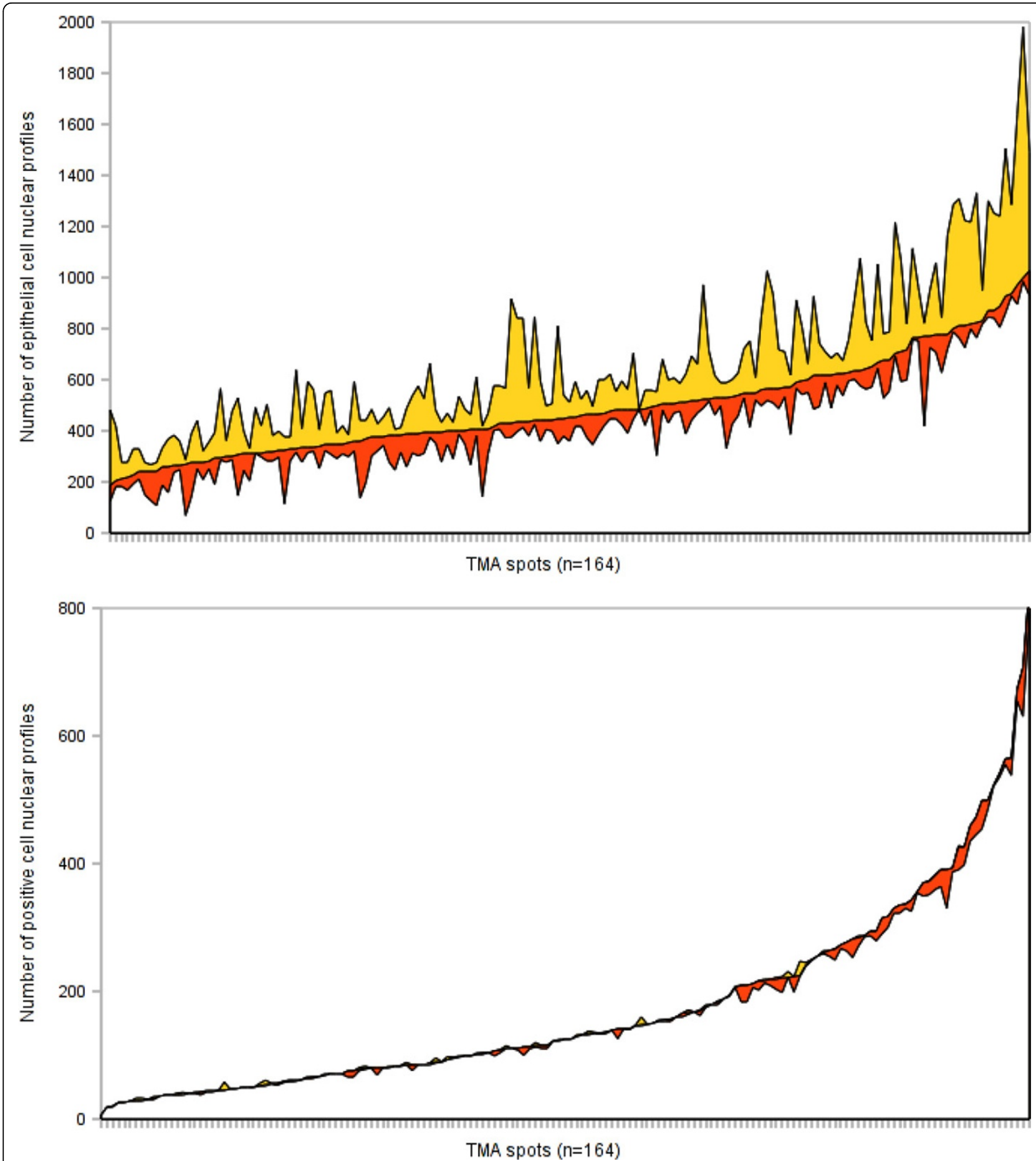

Figure 6 Upwards, the efforts of the experts for correcting undetected (yellow color area) and falsely detected (orange color area) epithelial cell nuclei and downwards, the efforts for correcting falsely labeled (yellow color area) and unlabeled nuclei (orange color area) for the whole TMA spot series, presented on the $x$ axis and sorted ascending by the value of the $Y$ axis.

\section{Conclusions}

We propose the dIHC Wizard to enable image analysisassisted stereology approach to produce reference data in a more efficient way. In our experiment, it reduced the expert manual work up to 3 times. Importantly, the tool can also support DIA quality control and improvement efforts since it provides detailed information on the accuracy of the DIA components applied - tumour 
tissue and tumour cell detection as well as interpretation of the Ki67 IHC positivity in the detected cells.

\section{List of abbreviations}

DIA: digital image analysis; IHC: immunohistochemistry; Ki67\%: the percentage of Ki67-positive tumor cells in each spot image; cD: corrected data; $\mathrm{dD}$ : digital data; $\mathrm{mD}$ : manual data; TMA: tissue microarray.

\section{Competing interests}

The authors declare that they have no competing interests.

\section{Authors' contributions}

BP designed and produced the software for the study, drafted the manuscript, performed statistical analyses. AL and PH drafted essential parts of the manuscript, participated in statistical analyses. Ail designed and carried out the digital image analyses, supervised the expert review procedures, edited the manuscript. AiL, IB, JB, RM performed the expert review. All authors participated in conception and design of the study, reviewing the analysis results, critically revised and approved the final manuscript.

\section{Authors' information}

None.

\section{Acknowledgements}

This research is funded by European Social Fund under the Global Grant measure.

Publication of this supplement has been funded by 12th European Congress on Digital Pathology. This article has been published as part of Diagnostic Pathology Volume 9 Supplement 1, 2014: Selected articles from the 12th European Congress on Digital Pathology. The full contents of the supplement are available online at http://www.diagnosticpathology.org/ supplements/9/S1

\section{Authors' details}

${ }^{1}$ Path-Image/BioTiCla, University of Normandy, Unicaen, Caen, France. ${ }^{2}$ Department of Pathology, Forensic Medicine and Pharmacology, Faculty of Medicine, Vilnius University, Vilnius, Lithuania. ${ }^{3}$ National Center of Pathology, affiliate of Vilnius University Hospital Santariskiu Clinics, Vilnius, Lithuania.

\section{Published: 19 December 2014}

\section{References}

1. Tadrous PJ: On the concept of objectivity in digital image analysis in pathology. Pathology 2010, 42:207-211.

2. Laurinavicius A, Laurinaviciene A, Dasevicius D, Elie N, Plancoulaine B, Bor C, Herlin P: Digital image analysis in pathology: benefits and obligation. Anal Cell Pathol (Amst) 2012, 35:75-78.

3. Brügmann A, Eld M, Lelkaitis G, Nielsen S, Grunkin M, Hansen JD, Foged NT, Vyberg M: Digital image analysis of membrane connectivity is a robust measure of HER2 immunostains. Breast Cancer Research and Treatment 2011.

4. Tang LH, Gonen M, Hedvat C, Modlin IM, Klimstra DS: Objective quantification of the Ki67 proliferative index in neuroendocrine tumors of the gastroenteropancreatic system: a comparison of digital image analysis with manual methods. Am J Surg Pathol 2012, 36:1761-1770.

5. Gudlaugsson E, Skaland I, Janssen EA, Smaaland R, Shao Z, Malpica A, Voorhorst F, Baak JP: Comparison of the effect of different techniques for measurement of Ki67 proliferation on reproducibility and prognosis prediction accuracy in breast cancer. Histopathology 2012, 61:1134-1144.

6. Laurinavicius A, Plancoulaine B, Laurinaviciene A, Herlin P, Meskauskas R, Baltrusaityte I, Besusparis J, Dasevi lus D, Elie N, labal Y, et al: A methodology to ensure and improve accuracy of Ki67 labelling index estimation by automated digital image analysis in breast cancer tissue. Breast Cancer Res 2014, 16:R35.

7. Ground-truth data cannot do it alone. Nature Methods 2011, 8:885-885.

8. Al-Kofahi Y, Lassoued W, Lee W, Roysam B: Improved Automatic Detection and Segmentation of Cell Nuclei in Histopathology Images. leee T BioMed Eng 2010, 57:841-852.
9. Bjornsson CS, Lin G, Al-Kofahi Y, Narayanaswamy A, Smith KL, Shain W, Roysam B: Associative image analysis: a method for automated quantification of 3D multi-parameter images of brain tissue. J Neurosci Methods 2008, 170:165-178.

10. Luisi J, Narayanaswamy A, Galbreath Z, Roysam B: The FARSIGHT Trace Editor: An Open Source Tool for 3-D Inspection and Efficient Pattern Analysis Aided Editing of Automated Neuronal Reconstructions. Neuroinformatics 2011, 9:305-315.

11. Laurinavicius A, Laurinaviciene A, Ostapenko V, Dasevicius D, Jarmalaite $S$, Lazutka J: Immunohistochemistry profiles of breast ductal carcinoma: factor analysis of digital image analysis data. Diagn Pathol 2012, 7:27.

12. Baddeley A, Jensen EBV: Stereology for statisticians Boca Raton, FL: Chapman \& Hall/CRC; 2005.

13. Bland JM, Altman DG: Measuring agreement in method comparison studies. Statistical methods in medical research 1999, 8:135-160.

14. Ljosa V, Sokolnicki KL, Carpenter AE: Annotated high-throughput microscopy image sets for validation. Nature Methods 2012, 9:637-637.

15. Peng HC, Roysam B, Ascoli GA: Automated image computing reshapes computational neuroscience. Bmc Bioinformatics 2013, 14.

doi:10.1186/1746-1596-9-S1-S8

Cite this article as: Plancoulaine et al:: Digital immunohistochemistry wizard: image analysis-assisted stereology tool to produce reference data set for calibration and quality control. Diagnostic Pathology 2014 9(Suppl 1):S8.

\section{Submit your next manuscript to BioMed Central and take full advantage of:}

- Convenient online submission

- Thorough peer review

- No space constraints or color figure charges

- Immediate publication on acceptance

- Inclusion in PubMed, CAS, Scopus and Google Scholar

- Research which is freely available for redistribution 